\title{
Controllable wave propagation of hybrid dispersive media with LC high-pass and band-pass networks
}

\author{
Edgar A. Flores Parra, ${ }^{1, a)}$ Andrea Bergamini, ${ }^{2}$ Bart Van Damme,${ }^{3}$ and Paolo Ermanni ${ }^{1}$ \\ ${ }^{1}$ Composite Materials and Adaptive Structures Laboratory, ETH Zürich, Leonhardstrasse 21, CH-8092 Zürich, \\ Switzerland \\ ${ }^{2}$ Empa, Materials Science and Technology, Laboratory for Mechanical Integrity of Energy Systems, \\ Überlandstrasse 129, CH-8600 Dübendorf, Switzerland \\ ${ }^{3}$ Empa, Materials Science and Technology, Laboratory for Acoustics and Noise Control, Überlandstrasse 129, \\ CH-8600 Dübendorf, Switzerland
}

(Received 10 February 2017; accepted 24 April 2017; published online 3 May 2017)

\begin{abstract}
This paper reports on the wave transmission characteristics of two configurations of a hybrid one dimensional (1D) medium. The hybrid characteristic is the result of the coupling between a 1D mechanical waveguide in the form of an elastic beam and an electrical network. The first configuration investigated is based on an LC high-pass network, while the second configuration is based on an LC band-pass network. For both networks, the capacitors are represented by a periodic array of piezoelectric elements that are bonded to the beam coupling, the mechanical and electrical domains, and thus the two waveguides. The coupling is characterized by a coincidence in frequency/wavenumber corresponding to the intersection of the dispersion curves. At this coincidence frequency, the hybrid medium features attenuation of wave motion as a result of the energy transfer to the electrical network. This energy exchange is depicted in the dispersion curves by eigenvalue crossing, a particular case of eigenvalue veering. This paper presents the numerical investigation of the wave propagation in the considered media along with experimental evidence of the wave transmission characteristics. The ability to conveniently tune the dispersion properties of the electrical network by varying the inductance is exploited to adapt the periodicity of the domain, i.e., monoatomic and diatomic unit cell configurations. Published by AIP Publishing.
\end{abstract}

[http://dx.doi.org/10.1063/1.4983088]

There has been increased interest in the control of elastic waves with arrays of periodic piezoelectric shunts for attenuation of mechanical vibrations. Most of the previous studies have focused on the reduction of structural vibrations with arrays of locally shunted piezoelectric elements ${ }^{1-3}$ or grounded interconnected piezoelectric elements. This paper reports on two interconnection schemes for the unit cell of periodic structures, the LC high-pass (HP) network and the LC band-pass network (BP). In Fig. 1(a), floating piezoelectric elements are interconnected in series using grounded inductors, thus forming a high-pass network. In Fig. 1(b), capacitors and inductors in parallel are connected in series by another set of inductors, yielding a band-pass network. As will be shown in this article, these extensions can have paramount effects on the overall dispersive properties of the resulting medium.

Forbidden frequency ranges in the dispersion curves of solid media through phononic crystals (PCs) and mechanical metamaterials (MMs) have been reported in the literature. In PCs, bandgaps result from periodic modulations of the mass density and/or elastic constants ${ }^{4,5}$ of the material resulting from the basis of the crystal (e.g., diatomic materials ${ }^{6}$ ). Such bandgaps exist for wavelengths on the order of the unit cell size and can be complete, ${ }^{7}$ namely, for any direction of propagation, or partial, which is direction specific. ${ }^{8}$ In metamaterials, on the other hand, the inclusion of suitably designed locally resonating units allows for the sub-wavelength

a)edgarf@ethz.ch modification of the dispersive properties of a medium, as reported among others by Liu in the mechanical domain. ${ }^{9}$ Waves at frequencies corresponding to wavelengths substantially larger than the unit cell size can be attenuated by local resonators. Moreover, while periodicity is not strictly necessary to achieve wave attenuation in correspondence with the tuning frequency of the resonator, ${ }^{10}$ it is often assumed to allow for the calculation of its properties and dispersion curves. In both PCs and MMs, as reported in the surveyed literature, waves propagate through the mechanical medium and interact with "inclusions" that either scatter them to

(a)
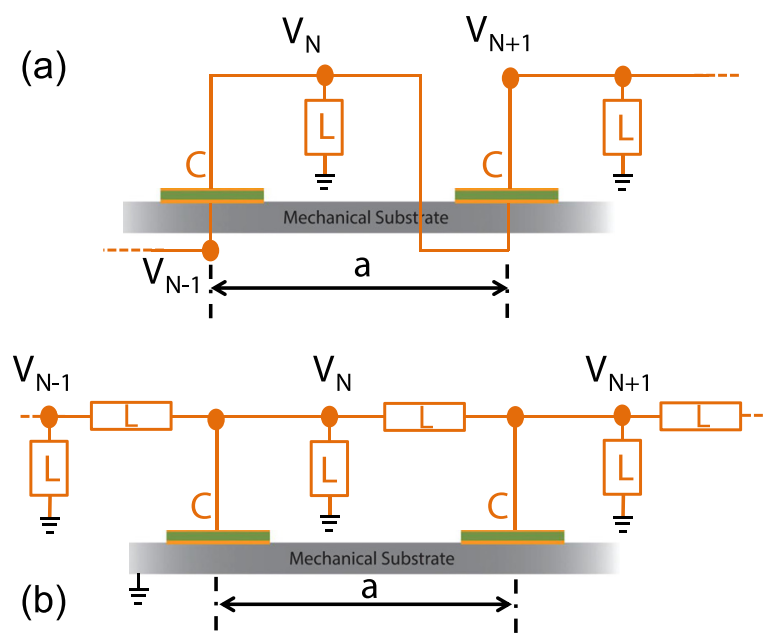

FIG. 1. (a) Unit cell HP and (b) unit cell BP. 
generate destructive interference at certain wavenumbers or absorb and dissipate energy through local resonances. In many of the reported materials, the nature of the inclusions is purely mechanical. ${ }^{7,9,11}$ In some cases, adaptive materials are exploited to modify the geometry of the unit cell, ${ }^{12}$ to tune the properties of the locally resonating units, ${ }^{13-15}$ or to modify the connectivity of a PC. ${ }^{16}$ In the latter cases, what could be defined as the electric domain of the unit cell is self-contained and only exchanges energy with the mechanical domain within the unit cell, and thus, it can be regarded as an inclusion in the mechanical medium. The mechanical component of the unit cell is thus the only pathway for the exchange of energy with neighboring cells.

Other interactions between mechanical and electrical modes can affect the propagation of waves, leading to attenuation. As discussed by Mace and Manconi, ${ }^{17}$ mode veering, and crossing, which can be considered a particular case of veering, occurs due to weak eigenvalue coupling, resulting in an exchange of energy between the modes. Crossing is the least common in the literature and is more often discussed along with the veering phenomena. At mode crossing, the two modes exist at the same frequency, and thus, the two modes are not uniquely defined and can be described as the resultant of the two independent eigenvectors approaching the crossing point. ${ }^{18}$

This work considers macroscopic media made of "artificial atoms" "19 that are made of hybrid assemblies. Such assemblies extend the functionality of the atoms by exploiting the electrical connectivity to allow simultaneous propagation of energy through the mechanical and electrical domains. The effect of interconnected piezoelectric shunts, to control the dynamic behavior of a structure, has also been explored by Dell'Isola et al., ${ }^{20-23}$ where multi-modal vibration damping is attained by tuning the electrical network to allow for the simultaneous internal resonance of all structural and electrical modes. Moreover, within the frame of interconnected uniform arrays of piezoelectric transducers coupled with electrical networks, Dell'Isola et al. ${ }^{24,25}$ have synthesized analog circuits with purely passive components to obtain efficient damping of mechanical vibrations in bars, beams, and plates.

In this contribution we will discuss the effects that the interactions between the electrical and mechanical modes have on the propagation of transverse mechanical waves, exploiting the proposed HP and BP networks of the hybrid medium.

The dispersion curves of the hybrid medium are calculated using numerical methods (FEM models implemented in COMSOL Multiphysics) by analyzing the eigenfrequencies of the unit cell modeled considering Floquet-Bloch boundary conditions. In the model of the one-dimensional hybrid medium, periodic boundary conditions are applied to obtain $u_{r}=u_{l} e^{-\mathrm{i} a k}$, where $u_{r}$ and $u_{l}$ are the mechanical degrees of freedom on the right and left sides of the unit cell, respectively. For the electrical network, periodicity is directly implemented using the Global ODEs and DAEs physics of COMSOL by imposing Eq. (5) in the case of the HP and Eq. (9) in the case of the BP. Eqs. (5) and (9) that characterize the HP and BP networks are derived by first relating the voltages $V_{N-1}$ and $V_{N+1}$ across the piezoelectric elements to the current at node $N . V_{N}$ and the voltages across the adjacent piezoelectric elements $V_{N-1}$ and $V_{N+1}$ are related through the Floquet-Bloch boundary conditions given by Eqs. (1) and (2). For the HP, the relationship between voltage and current in the unit cell leads to Eq. (3), while for the $\mathrm{BP}$, it leads to Eq. (8). $L$ is the value of the inductor in the unit cell, $k$ is the wavenumber, and $a$ is the lattice constant of the medium. Finally, the voltage $V_{N}$ is related to the charge $Q$ on the top electrode of the piezoelectric element by $V_{N}=Q / C$, where $C$ is the capacitance of the piezoelectric element. Time, $t$, is taken into account assuming harmonic oscillating charges $(Q=q \sin (\omega t))$.

The transmittance calculations of the HP and BP hybrid media shown in Figs. 4(b)-6(b) are modeled using COMSOL Multiphysics. The dimension of the modeled beam is $1 \mathrm{~mm} \times 7 \mathrm{~mm} \times 500 \mathrm{~mm}$. Along the center portion of the structure, the hybrid medium with a unit cell size of $10 \mathrm{~mm}$ is added. For the HP configuration, 17 unit cells are positioned between $150 \mathrm{~mm}$ and $320 \mathrm{~mm}$ as shown in Fig. 2, whereas for the BP configuration, 15 unit cells from either side of the beam are positioned between $150 \mathrm{~mm}$ and $300 \mathrm{~mm}$. The beam material is 6061 aluminum alloy, while the piezoelectric element material is STEMiNC, SM111. The piezoelectric elements have a diameter of $d=7 \mathrm{~mm}$ and a thickness of $t_{p}=0.4 \mathrm{~mm}$, yielding a capacitance value of around $C=850 \mathrm{pF}$. The inductive and resistive components are modeled using the electric circuit physics of COMSOL. The mechanical transmittance of the finite hybrid medium is calculated by taking the ratio of the spatial average of the velocity amplitudes, over a region with $100 \mathrm{~mm}$ in length, before and after the periodic arrangement.

The experimental set-ups for the HP and BP configurations, shown in Fig. 2, were implemented using real inductors. The monoatomic configuration of both the HP and BP used an inductance $L=100 \mathrm{mH}$. The diatomic configuration of the HP used a combination of $L_{1}=100 \mathrm{mH}$ and $L_{2}=15 \mathrm{mH}$. All electrical components were arranged on a breadboard to facilitate reconfiguring the connections.

$$
\begin{gathered}
V_{N-1}=V_{N} e^{\mathrm{i} a k}, \\
V_{N+1}=V_{N} e^{-\mathrm{i} a k}, \\
C\left(\dot{V}_{N-1}-\dot{V}_{N+1}-2 \dot{V}_{N}\right)-\int \frac{V_{N}}{L} d t=0, \\
V_{N}=Q / C, \\
L C \ddot{Q}\left(e^{-\mathrm{i} a k}+e^{\mathrm{i} a k}-2\right)-Q=0 .
\end{gathered}
$$

The wave attenuation capabilities of the HP hybrid medium can be further exploited by introducing a diatomic unit cell configuration. A diatomic unit cell can be designed by alternating both the capacitance or inductance values.

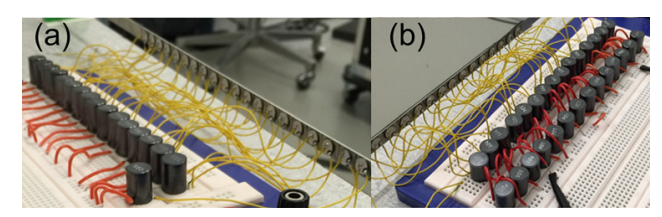

FIG. 2. (a) Experimental setup for HP and (b) experimental setup for BP. 
In this case, a diatomic inductance configuration, described by Eqs. (6) and (7), was investigated, yielding the dispersion curves shown in Fig. 5(a).

$$
\begin{gathered}
\ddot{Q}_{1}\left(e^{\mathrm{i} a k}+1\right)-2 \ddot{Q}_{2}-Q_{2} /\left(L_{1} C\right)=0, \\
\ddot{Q}_{2}\left(e^{-\mathrm{i} a k}+1\right)-2 \ddot{Q}_{1}-Q_{1} /\left(L_{2} C\right)=0 .
\end{gathered}
$$

The BP network is the second configuration for controlling the wave attenuation capabilities of the hybrid medium. The BP monoatomic unit cell configuration is described by Eq. (9). The BP dispersion curves are displayed in Figs. 3(c) and 6(a).

$$
\begin{gathered}
\frac{1}{L} \int\left(V_{N-1}+V_{N+1}-3 V_{N}\right) d t-C \dot{V}_{N}=0, \\
L C \ddot{Q}-Q\left(e^{-\mathrm{i} a k}+e^{\mathrm{i} a k}-3\right)=0 .
\end{gathered}
$$

The dispersion of the HP electrical mode, Fig. 4(a), shows a medium where the phase velocity is positive while the group velocity is negative, yielding a backward wave phenomenon. As shown in Figs. 4(a)-6(a), eigenvalue crossing occurs at the intersections between the electrical and transverse mechanical modes for both the HP and BP. It is at the frequencies and wavenumber corresponding to crossing that attenuation occurs, indicating an exchange of energy between the mechanical and electrical domains.

Moreover, due to the shape of the HP electrical mode, attenuation at lower frequencies can be achieved with smaller inductances than for other network configurations, i.e., for a capacitance of $850 \mathrm{pF}$ and $L=100 \mathrm{mH}$, the highpass configuration can attenuate frequencies around $10 \mathrm{kHz}$. Conversely, a low-pass filter ${ }^{26}$ with an equivalent piezoelectric capacitance would require inductances around $0.8 \mathrm{H}$. Likewise, if local resonator shunts were implemented, inductances of around $0.3 \mathrm{H}$ would be required for a frequency of $10 \mathrm{kHz}, f_{c}=1 /(2 \pi \sqrt{L C})$. Fig. 3 shows that two attenuation zones can be implemented with a diatomic HP unit cell. The
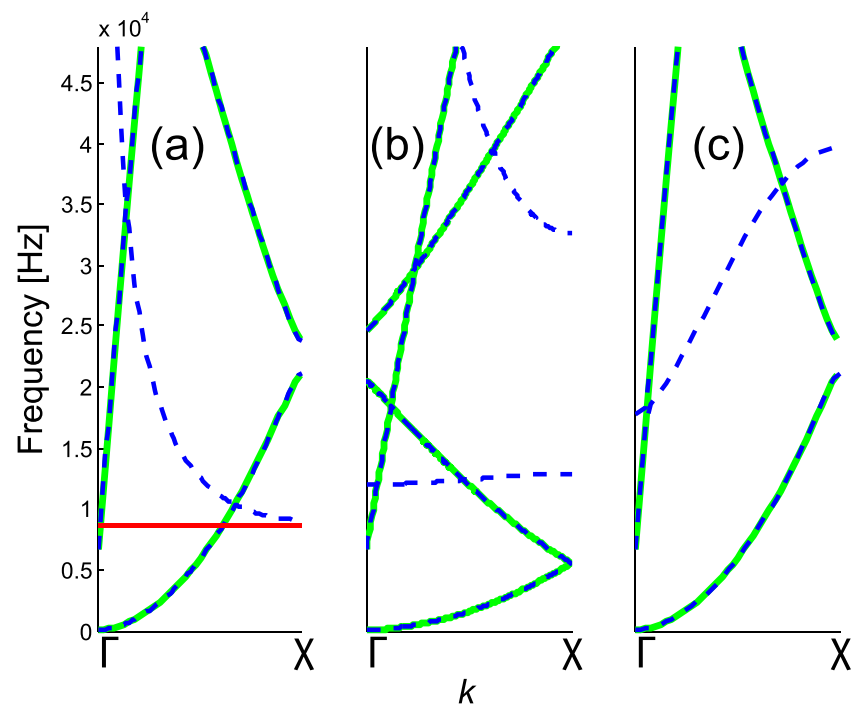

FIG. 3. (a) HP monoatomic dispersion for $L=100 \mathrm{mH}$, where the cut-off frequency of the LC high-pass network is given by $f_{h p}=1 /(4 \pi \sqrt{L C})$ and indicated by the red line. (b) Diatomic dispersion for $L_{1}=100 \mathrm{mH}$ and $L_{2}=15 \mathrm{mH}$. (c) BP monoatomic dispersion for $L=100 \mathrm{mH}$.
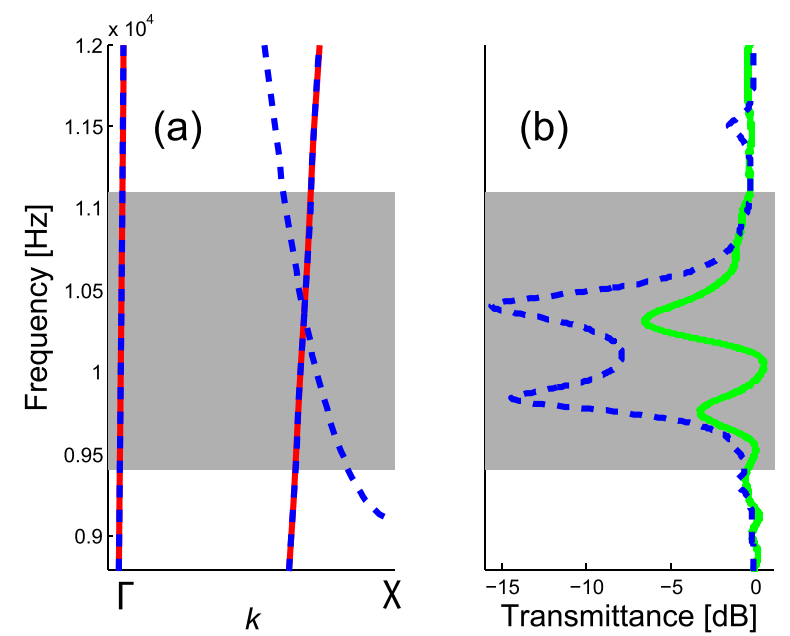

FIG. 4. (a) Mode crossing for monoatomic dispersion of HP with purely mechanical modes (solid red) and coupled modes (dotted blue). (b) Numerical (dotted blue) and experimental (solid green) transmittance curves of the hybrid media for $L=100 \mathrm{mH}$.

diatomic configuration allows for tuning of two independent attenuation zones using the same network. In the dispersion curves, the diatomic HP configuration is interpreted as the existence of two electrical modes. The higher frequency electrical mode has the same shape as that of the monoatomic HP configuration, while the electrical mode at the lower frequency as shown in Fig. 5(a) has an $S$ shape resembling that of a BP electrical mode ${ }^{27}$ shown in Fig. 1(c). Fig. 1 (c) shows the dispersion curves of the BP network which is characterized by an $S$ shape electrical mode. As shown in Fig. 6(a), just like the HP network, the BP electrical mode crosses the transverse mechanical mode, leading to wave attenuation. However, for the same inductance as the HP network, $L=100 \mathrm{mH}$, the BP network displays crossing at a much higher frequency (around $37 \mathrm{kHz}$ ). The BP configuration offers the advantage of a banded electrical mode contained between lower and upper frequencies, $f_{l}=1$ / $(2 \pi \sqrt{L C}) f_{u}=5 /(2 \pi \sqrt{L C})$, whereas the HP displays an asymptotic behavior for the decreasing wavenumber.
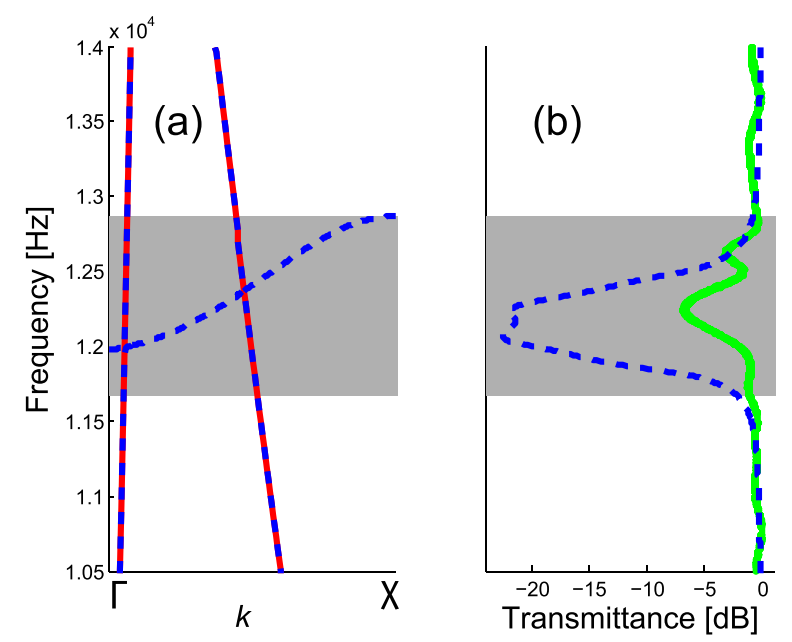

FIG. 5. (a) Mode crossing dispersion of the first electrical mode of diatomic configuration and (b) numerical (dotted) and experimental (solid) transmittance curves of the media for $L_{1}=100 \mathrm{mH}$ and $L_{2}=15 \mathrm{mH}$. 

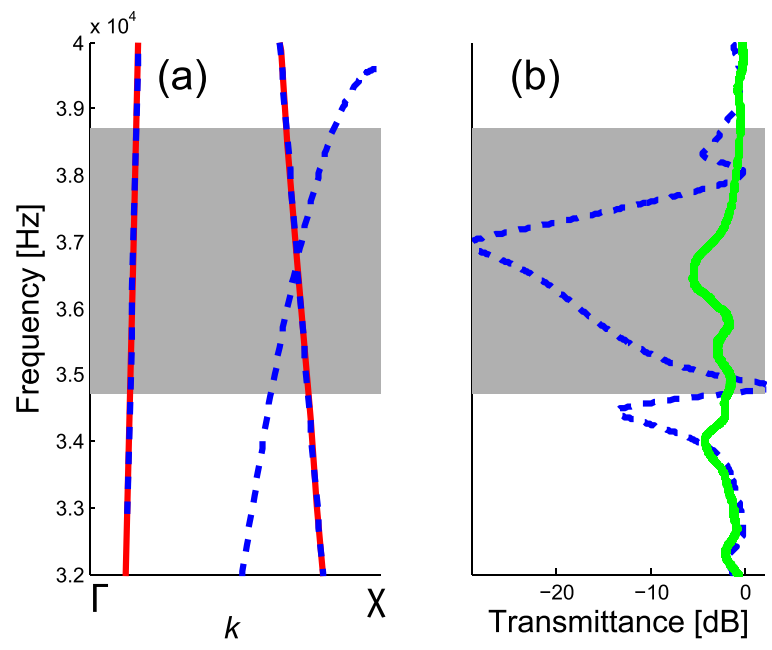

FIG. 6. (a) Mode crossing for monoatomic dispersion of BP with purely mechanical modes (solid red) and coupled modes (dotted blue). (b) Numerical (dotted) and experimental (solid green) transmittance curves of the media for $L=100 \mathrm{mH}$.

In the present work, we have shown the versatility of two different interconnection schemes for generating areas of low mechanical transmittance by introducing two networks through which energy can propagate, thereby attenuating transverse waves in their mechanical counterpart at the eigenvalue crossing frequencies. The rationale behind introducing coupled electrical networks lies in the ability to shape a system mechanical response (characterized by its dispersion properties) without changing the mechanical layout. The HP offers an implementation where the piezoelectric elements do not need to be grounded and requires significantly smaller inductance values to achieve attenuation at the same frequency as the low-pass filter, ${ }^{26}$ the local resonant shunts, or the BP configuration. The HP diatomic configuration leads to two independent attenuation regions characterized by two separate electrical modes, one of which resembles the electrical mode of the BP but over a smaller frequency range. The BP yields a bounded electrical mode that can also lead to attenuation due to eigenvalue crossing. By coupling a mechanical system to different electrical networks, we have shown that the dispersion properties of the hybrid medium can be tailored to achieve mechanical wave attenuation at one or multiple desired frequencies.
This research was funded by the Swiss National Science Foundation (SNSF, Grant No. 200021_157060) and the Mexican National Science Foundation (CONACYT).

${ }^{1}$ J. J. Hollkamp, J. Intell. Mater. Syst. Struct. 5, 49 (1994).

${ }^{2}$ B. S. Beck, K. A. Cunefare, M. Ruzzene, and M. Collet, J. Intell. Mater. Syst. Struct. 5, 1177 (2011).

${ }^{3}$ A. Spadoni, M. Ruzzene, and K. Cunefare, J. Intell. Mater. Syst. Struct. 20, 979 (2009).

${ }^{4}$ L. Brillouin, Wave Propagation in Periodic Structures: Electric Filters and Crystal Lattices (Courier Corporation, 1946).

${ }^{5}$ P. A. Deymier, Acoustic Metamaterials and Phononic Crystals (Springer Science \& Business Media, 2013), Vol. 173.

${ }^{6}$ S. Krödel, T. Delpero, A. Bergamini, P. Ermanni, and D. M. Kochmann, Adv. Eng. Mater. 16, 357 (2014).

${ }^{7}$ T.-T. Wu, Z.-G. Huang, T.-C. Tsai, and T.-C. Wu, Appl. Phys. Lett. 93, 111902 (2008).

${ }^{8}$ A. Sato, Y. Pennec, T. Yanagishita, H. Masuda, W. Knoll, B. DjafariRouhani, and G. Fytas, New J. Phys. 14, 113032 (2012).

${ }^{9}$ Z. Liu, X. Zhang, Y. Mao, Y. Zhu, Z. Yang, C. Chan, and P. Sheng, Science 289, 1734 (2000).

${ }^{10}$ H. Huang and C. Sun, Philos. Mag. 91, 981 (2011).

${ }^{11}$ J.-H. Lee, J. P. Singer, and E. L. Thomas, Adv. Mater. 24, 4782 (2012).

${ }^{12}$ J.-F. Robillard, O. B. Matar, J. Vasseur, P. A. Deymier, M. Stippinger, A.-C. Hladky-Hennion, Y. Pennec, and B. Djafari-Rouhani, Appl. Phys. Lett. 95, 124104 (2009).

${ }^{13}$ L. Airoldi and M. Ruzzene, J. Intell. Mater. Syst. Struct. 22, 1567 (2011).

${ }^{14}$ F. Casadei, T. Delpero, A. Bergamini, P. Ermanni, and M. Ruzzene, J. Appl. Phys. 112, 064902 (2012).

${ }^{15}$ M. Collet, K. A. Cunefare, and M. N. Ichchou, J. Intell. Mater. Syst. Struct. 20, 787 (2009).

${ }^{16}$ A. Bergamini, T. Delpero, L. D. Simoni, L. D. Lillo, M. Ruzzene, and P. Ermanni, Adv. Mater. 26, 1343 (2014).

${ }^{17}$ B. R. Mace and E. Manconi, J. Acoust. Soc. Am. 131, 1015 (2012).

${ }^{18} \mathrm{G}$. Kerschen, in Conference Proceedings of the Society for Experimental Mechanics Series (Springer, Berlin, 2014).

${ }^{19}$ M. Lapine and S. Tretyakov, IET Microwaves, Antennas Propag. 1, 3 (2007).

${ }^{20}$ R. C. Batra, F. Dell'Isola, S. Vidoli, and D. Vigilante, Int. J. Solids Struct. 42, 3115 (2005).

${ }^{21}$ C. Maurini, F. Dell'Isola, and D. Del Vescovo, Mech. Syst. Signal Process. 18, 1243 (2004).

${ }^{22}$ F. Dell'Isola, C. Maurini, and M. Porfiri, Smart Mater. Struct. 13, 299 (2004).

${ }^{23}$ B. Lossouarn, M. Aucejo, and J. Deü, Smart Mater. Struct. 24, 045018 (2015).

${ }^{24}$ F. Dell'Isola, M. Porfiri, and S. Vidoli, C. R. Mec. 331, 69 (2003).

${ }^{25}$ S. Alessandroni, F. Dell'Isola, and M. Porfiri, Int. J. Solids Struct. 39, 5295 (2002).

${ }^{26}$ A. E. Bergamini, M. Zündel, E. A. F. Parra, T. Delpero, M. Ruzzene, and P. Ermanni, J. Appl. Phys. 118, 154310 (2015).

${ }^{27} \mathrm{~F}$. Martín, Artificial Transmission Lines for $R F$ and Microwave Applications (John Wiley \& Sons Inc., 2015). 